\title{
Engineering Model of High Pressure Moist Air
}

\author{
Tomáš Hyhlík ${ }^{1, \star}$ \\ ${ }^{1}$ CTU in Prague, FME, Department of Fluid Dynamics and Thermodynamics, Technická 4, 16607 Prague
}

\begin{abstract}
The article deals with the moist air equation of state. There are equations of state discussed in the article, i.e. the model of an ideal mixture of ideal gases, the model of an ideal mixture of real gases and the model based on the virial equation of state. The evaluation of sound speed based on the ideal mixture concept is mentioned. The sound speed calculated by the model of an ideal mixture of ideal gases is compared with the sound speed calculated by using the model based on the concept of an ideal mixture of real gases. The comparison of enthalpy end entropy based on the model of an ideal mixture of ideal gases and the model of an ideal mixture of real gases is performed. It is shown that the model of an ideal mixture of real gases deviates from the model of an ideal mixture of ideal gases only in the case of high pressure. An impossibility of the definition of partial pressure in the mixture of real gases is discussed, where the virial equation of state is used.
\end{abstract}

\section{Basic parameters of moist air}

This section covers only a few selected parameters of moist air. The detailed description can be found e.g. in [1]. Let us consider the moist as a binary mixture of dry air and water vapour. An amount of water vapour can change from zero (it is dry air) to some maximum, which depends on the temperature and pressure. Humidity ratio is defined as the ratio of mass of water vapour to the mass of dry air

$$
x=\frac{V \rho_{v}}{V \rho_{a}}=\frac{\rho_{v}}{\rho_{a}},
$$

where $V$ is volume, $\rho_{v}$ is water vapour density (absolute humidity) and $\rho_{a}$ is dry air density. Let $x^{\prime \prime}$ is the humidity ratio of saturated moist air at the same temperature $t$ and pressure $p$. Degree of saturation is defined as the ratio of air humidity ratio $x$ to humidity ratio of saturated moist air $x^{\prime \prime}$

$$
\mu=\left(\frac{x}{x^{\prime \prime}}\right)_{p, t} .
$$

The value of saturated humidity ratio $x^{\prime \prime}$ is limited in the atmospheric moist air, e.g. that is in the case of the pressure $p=98000 \mathrm{~Pa}$ and temperature $t=25^{\circ} \mathrm{C}$. There can by a different story in some technical application. Consider the case of the same pressure $p=98000 \mathrm{~Pa}$ and at the temperature $t=150^{\circ} \mathrm{C}$, where is water vapour always superheated for arbitrary vapour pressure $p_{v} \leq p$, therefore moist air can not be saturated for arbitrary humidity ratio $x$. In such a situation is appropriate to define $x^{\prime \prime}=\infty$ and degree of saturation will be zero.

Relative humidity $\varphi_{R}$ is frequently used parameter in psychrometrics. Relative humidity is defined as the ratio of absolute humidity $\rho_{v}$ to absolute humidity of saturated

\footnotetext{
^e-mail: tomas.hyhlik@fs.cvut.cz
}

moist air $\rho_{v}^{\prime \prime}$ at the same temperature $t$ and pressure $p$

$$
\varphi_{R}=\left(\frac{\rho_{v}}{\rho_{v}^{\prime \prime}}\right)_{p, t} .
$$

Absolute humidity of moist air $\rho_{v}$ expresses weight of water vapour in cubic meter of moist air.

The partial pressure of water vapour in the saturated moist air is not in general equal to saturated water vapour pressure $p_{\text {sat }}$ at given temperature, which is related to pure water vapour [2]. Partial pressure of water vapour is

$$
p_{v}=f_{e} p_{s a t},
$$

where $f_{e}$ is so called enhancement factor, which has the value of 1.0041 at standard atmospheric pressure $101325 \mathrm{~Pa}$ and at temperature $0^{\circ} \mathrm{C}$. In general, it is possible to say that the value of enhancement factor is going closer to one with increasing temperature and with decreasing temperature its value increasing. The value of enhancement factor is 1.46383 at $10 \mathrm{MPa}$ a $0^{\circ} \mathrm{C}$, and after temperature increases to $200^{\circ} \mathrm{C}$ is its value 1.21286 [2].

\section{Model of ideal mixture of ideal gases}

The most simplest moist air model is the model of ideal mixture of ideal gases. The pressure can be in ideal mixture expressed by using Dalton's law

$$
p=p_{a}+p_{v}
$$

where $p_{a}$ is partial pressure of dry air and $p_{v}$ is partial pressure of water vapour. Partial pressure is hypothetical pressure of gas if it alone occupied the volume of the mixture at the same temperature. An individual components of ideal mixture exist independently without any interaction. The 
moist air density is in the ideal mixture expressed as a sum of individual partial densities

$$
\rho=\rho_{a}+\rho_{v}
$$

Partial densities can be expressed by using ideal gas equation of state as

$$
\rho_{a}=\frac{p_{a} M_{a}}{R T}, \quad \rho_{v}=\frac{p_{v} M_{v}}{R T} .
$$

Mass fractions of dry air and water vapour are

$$
w_{a}=\frac{\rho_{a}}{\rho}, \quad w_{v}=\frac{\rho_{v}}{\rho},
$$

where

$$
w_{a}+w_{v}=1 .
$$

Mass fractions can be expressed using humidity ratio

$$
w_{a}=\frac{1}{1+x}, \quad w_{v}=\frac{x}{1+x} .
$$

The moist air equation of state of the mixture of dry air and water vapour can be derived using Dalton's law (5), the definition of humidity ratio (1) and the relation for moist air density (6) as

$$
\rho=\frac{p}{R T} \frac{M_{a}(1+x)}{\left(1+\frac{M_{a}}{M_{v}} x\right)},
$$

where $M_{a}$ is molecular weight of dry air, $M_{v}$ is molecular weight of water vapour, $R$ is universal gas constant and $T$ is temperature.

To determine enthalpy and entropy it is necessary to choose reference state, which will determine zero value of that parameters. An appropriate choice is e.g. triple point of water, where $T_{\text {ref }}=273.16 \mathrm{~K}, p_{\text {ref }}=611.657 \mathrm{~Pa}$. Enthalpy per kilogramme of mixture is expressed as

$$
h=w_{a} c_{p_{a}}\left(T-T_{r e f}\right)+w_{v}\left[c_{p_{v}}\left(T-T_{r e f}\right)+l_{r e f}\right],
$$

where $l_{\text {ref }}$ is specific latent heat at reference point, specific heat capacity of dry air $c_{p_{a}}$ and specific heat capacity of water vapour $c_{p_{v}}$ are expressed as

$$
c_{p_{a}}=\frac{\kappa_{a}}{\kappa_{a}-1} r_{a}, \quad c_{p_{v}}=\frac{\kappa_{v}}{\kappa_{v}-1} r_{v} .
$$

Specific gas constant of dry air $r_{a}$ and water vapour $r_{v}$ are

$$
r_{a}=\frac{R}{M_{a}}, \quad r_{v}=\frac{R}{M_{v}} .
$$

Poison's coefficient of dry air is $\kappa_{a}=1.4$ and in the case of water vapour is $\kappa_{v}=1.33$. Enthalpy is usually introduced in psychrometrics as enthalpy per kilogramme of dry air [1]

$$
h_{1+x}=c_{p_{a}}\left(T-T_{r e f}\right)+x\left[c_{p_{v}}\left(T-T_{r e f}\right)+l_{r e f}\right],
$$

where the first part is related to dry air and the second part is related to water vapour, where there is $x$ kilograms of water vapour in one kilogram of dry air, and therefore is the moist air total enthalpy $H$ obtained by multiplying of $h_{1+x}$ with the weight of dry air in the mixture dry air and water vapour.

To determine specific entropy per kilogram of mixture, which is defined as

$$
s=w_{a} s_{a}+w_{v} s_{v},
$$

is necessary to express partial entropies of individual components of mixture. Specific entropy of dry air is

$$
s_{a}=c_{p_{a}} \ln \left(\frac{T}{T_{r e f}}\right)-\frac{R}{M_{a}} \ln \left(\frac{p_{a}}{p_{r e f}}\right)
$$

and water vapour specific entropy is similarly

$$
s_{v}=c_{p_{v}} \ln \left(\frac{T}{T_{r e f}}\right)-\frac{R}{M_{v}} \ln \left(\frac{p_{v}}{p_{r e f}}\right) .
$$

Speed of sound can be in the case of ideal mixture of ideal gases expressed by using its definition and finally by the relation for ideal gases

$$
a=\sqrt{\left(\frac{\partial p}{\partial \rho}\right)_{s}}=\sqrt{\kappa r T} .
$$

Specific gas constant of the mixture can be expressed using gas constants of individual components

$$
r=w_{a} r_{a}+w_{v} r_{v}
$$

The Poisson exponent of the mixture is

$$
\kappa=\frac{c_{p}}{c_{v}}
$$

where individual heat capacities are

$$
c_{p}=w_{a} c_{p_{a}}+w_{v} c_{p_{v}}, \quad c_{v}=c_{p}-r .
$$

\section{Advanced engineering model}

The advanced engineering model is the model of an ideal mixture of real gases, and therefore is based on the Dalton's law (5). To determine the moist air properties it is necessary to get partial pressures and partial densities $p_{a}$, $p_{v}, \rho_{a}$ a $\rho_{v}$ from the given parameters of moist air, and these are namely the moist air temperature $T$, pressure $p$ and humidity ratio $x$. The problem contains four unknowns, which can be obtained using the solution of the system of algebraic equations (5), (1),

$$
p-p_{v}=p_{a i r}\left(T, \rho_{a}\right)
$$

and

$$
p-p_{a}=p_{v a p}\left(T, \rho_{v}\right),
$$

where $p_{\text {air }}\left(T, \rho_{a}\right)$ represents dry air equation of state e.g. according to [3] and $p_{v a p}\left(T, \rho_{v}\right)$ represents water vapour equation of state e.g. according to [4]. The real gases equations of state are used to solve pressure depending on the temperature and the density. The moist air density is calculated using equation (6), where partial densities are 
used. The real gas equations of state [3, 4] allow calculating enthalpy and entropy of dry air

$$
h_{a}=h_{a i r}\left(T, \rho_{a}\right), \quad s_{a}=s_{a i r}\left(T, \rho_{a}\right)
$$

and enthalpy and entropy of water vapour

$$
h_{v}=h_{v a p}\left(T, \rho_{v}\right), \quad s_{v}=s_{v a p}\left(T, \rho_{v}\right) .
$$

Moist air entropy is solved using equation (16). Enthalpy is

$$
h=w_{a} h_{a}+w_{v} h_{v} .
$$

The speed of sound of individual components of the mixture can be evaluated using real gas equations of state $[3,4]$

$$
a_{a}=a_{a i r}\left(T, \rho_{a}\right), \quad a_{v}=a_{v a p}\left(T, \rho_{v}\right) .
$$

Reference [5] offers possibility to evaluate speed of sound using relation for ideal mixture

$$
a=\frac{1}{\sqrt{M\left(\frac{\psi_{v}}{M_{v} a_{v}^{2}}+\frac{\psi_{a}}{M_{a} a_{a}^{2}}\right)}}
$$

where mole fractions are

$$
\psi_{a}=\frac{n_{a}}{n}, \quad \psi_{v}=\frac{n_{v}}{n}
$$

and molar mass of moist air is

$$
M=\psi_{a} M_{a}+\psi_{v} M_{v}
$$

Molar fractions and mass fractions are related through the ratio of molecular weights

$$
w_{a}=\frac{M_{a}}{M} \psi_{a}, \quad w_{v}=\frac{M_{v}}{M} \psi_{v} .
$$

Equation of state of the real gas is possible to assume in the form

$$
p V=n R T z(p, T),
$$

where $z(p, T)$ is compressibility factor. It is possible to express equations of state for dry air and water vapour as

$$
p_{a} V=n_{a} R T z_{a}\left(p_{a}, T\right), \quad p_{v} V=n_{v} R T z_{v}\left(p_{v}, T\right) .
$$

After addition of equations (34) and comparison with equation (33) is possible to express compressibility factor of the mixture as

$$
z(p, T)=\psi_{a} z_{a}\left(p_{a}, T\right)+\psi_{v} z_{v}\left(p_{v}, T\right) .
$$

It means, that the consequence of utilising the Dalton's law for the mixture of real gases is, that compressibility factor of the mixture of real gases is the weighted average of individual compressibility factors [6].

\section{Virial equation of state}

Compressibility factor is possible to define in the real gas as

$$
z=\frac{p V_{m}}{R T}
$$

where $V_{m}$ is molar volume

$$
V_{m}=\frac{V}{n}=\frac{V}{n_{a}+n_{v}} .
$$

Reference [7] shows, that it is possible to express compressibility factor by using power series depending on the molar concentration $n / V=1 / V_{m}$

$$
z=1+B\left(\frac{1}{V_{m}}\right)+C\left(\frac{1}{V_{m}}\right)^{2}+\ldots,
$$

where $B(T)$ is the second and $C(T)$ is the third virial coefficient. The second virial coefficient of the mixture is possible to express according to [7] as

$$
B=\psi_{a}^{2} B_{a}+\psi_{v}^{2} B_{v}+2 \psi_{a} \psi_{v} B_{a v}
$$

where $B_{a}$ and $B_{v}$ are the second virial coefficients of the pure components and $B_{a v}$ is the second virial coefficient which is connected with the interaction of dry air and water vapour. The relations for individual components are

$$
p_{a} V=n_{a} R T+\frac{n_{a}^{2} R T B_{a}}{V}, \quad p_{v} V=n_{v} R T+\frac{n_{v}^{2} R T B_{v}}{V} .
$$

If the Dalton's law is valid, then

$$
p V=\left(n_{a}+n_{v}\right) R T+\frac{R T}{V}\left(n_{a}^{2} B_{a}+n_{v}^{2} B_{v}\right)
$$

and after manipulation is

$$
\frac{p V_{m}}{R T}=1+\left(\psi_{a}^{2} B_{a}+\psi_{v}^{2} B_{v}\right) \frac{1}{V_{m}} .
$$

From the comparison of equation (42) with equations (39) and (38) is evident, that Dalton's law ignored molecular interaction [6]. If the virial equation of state is applied to the mixture is not Dalton's law taken into account and partial pressures are not clearly defined. Determination of partial pressures is possible by using molar fractions like when it is the mixture of ideal gases, where the molar fractions are determined using fractions of partial pressure and the mixture pressure

$$
p_{a}^{A}=\psi_{a} p, \quad p_{v}^{A}=\psi_{v} p .
$$

Determination of partial pressures is possible using classical definition, where it is the pressure of the mixture component at temperature $T$ occupying the overall volume $V$. From the equations of state for individual components (40) is

$$
p_{a}^{B}=\frac{n_{a} R T}{V}+\frac{n_{a}^{2} R T B_{a}}{V^{2}}, \quad p_{v}^{B}=\frac{n_{v} R T}{V}+\frac{n_{v}^{2} R T B_{v}}{V^{2}},
$$

or by using of the mixture molar volume is

$p_{a}^{B}=\psi_{a}\left[\frac{R T}{V_{m}}\left(1+\psi_{a} \frac{B_{a}}{V_{m}}\right)\right], \quad p_{v}^{B}=\psi_{v}\left[\frac{R T}{V_{m}}\left(1+\psi_{v} \frac{B_{v}}{V_{m}}\right)\right]$.

It is clear, that square bracket in equations (45) does not correspond to the pressure of the mixture according to (38) 
and (39). Partial pressures based on both definitions are not in general equal

$$
p_{a}^{A} \neq p_{a}^{B}, \quad p_{v}^{A} \neq p_{v}^{B} .
$$

They agree only in the case of the ideal gas equation of state, where are virial coefficients zero.

Nowadays virial equation of state is standard in psychrometrics [2]. Virial coefficients are based on the general equation for water vapour according to [4] and on the general equation of state for dry air according to [3]. Equation of state according to [2] is based on the second and on the third virial coefficients

$$
p\left(V_{m}, T, \psi_{a}\right)=\frac{R T}{V_{m}}\left(1+\frac{B\left(\psi_{a}, T\right)}{V_{m}}+\frac{C\left(\psi_{a}, T\right)}{V_{m}^{2}}\right),
$$

where for given pressure $p$, temperature $T$ and molar fraction of dry air $\psi_{a}$ is possible to solve iteratively molar volume $V_{m}$, and finally is the mixture density evaluated using molecular weight of moist air $M$ as

$$
\rho=\frac{M}{V_{m}} .
$$

Pressure is possible to solve directly in the case, where temperature $T$, density $\rho$ and molar fraction of dry air $\psi_{a}$ are given. The last possibility is an iterative solution of temperature $T$ as a function of the density $\rho$, pressure $p$ and dry air molar fraction. The equation of state according to [2] allows solving also other state variables like enthalpy $h$ and entropy $s$.

\section{Results}

The comparison of the model of an ideal mixture of ideal gases and the model of an ideal mixture of real gases is depicted in the figures. Figures 1 and 2 shows the dependence of enthalpy and entropy on the pressure and on the degree of saturation. It is visible, that even a minimal amount of water vapour influence at a high temperature significantly the values of enthalpy and entropy. It is clear, that both compared models give practically the same results at low pressures. There are significant discrepancies at a hight value of pressure such as the pressure $5 \mathrm{MPa}$ and $10 \mathrm{MPa}$. Figure 3 documents deviations in the prediction of water vapour amount in the moist air, which is based on the both compared models. There are significant deviations at the pressure of $5 \mathrm{MPa}$ and $10 \mathrm{MPa}$ similarly like in the cases of enthalpy and entropy. Figure 4 shows the density comparison calculated by using tested models. It is possible to see, that there are significant discrepancies not only at a high pressure but also at a high value of temperatures. There is a high value of humidity ratio at high temperatures, and therefore it is possible to explain discrepancies using the inappropriate ideal gas model. There is a relation of the speed of sound on the pressure and the temperature in figure 4 . It is clear from the figure, that the model of ideal gas mixture does not take into consideration the effect of pressure on the speed of sound. It is clear, that the model of an ideal mixture of ideal gases can be used at low pressures, but at the pressure of $1 \mathrm{MPa}$ are for the high value of humidity ratio evident discrepancies.

\section{Conclusions}

The model of an ideal mixture of ideal gases is applicable predominantly in the case of atmospheric and lower pressures such as in the case of evaporative cooling [8, 9]. The moist air model based on the virial equation of state is applicable in a wider range of pressures and temperatures, how is stated in [2]. The limit of applicability of this model can be maximal humidity ratio, which is according to [2] $10 \mathrm{~kg}_{\mathrm{p}} / \mathrm{kg}_{\mathrm{sv}}$. The model of an ideal mixture of real gases is in the range of validity of virial equation of state less accurate, but there is an advantage out of the range of its validity, where the real gas effect is taken into account. An advantage of this model can be seen e.g. in the case of a very high value of the water vapour mass fraction. There can be a problem with the speed of sound calculation because reference [2] does not contain any relevant equation, but the concept based on ideal mixture rule (29) is presented in this article.

\section{Acknowledgement}

This work has been supported by Technology Agency of the Czech Republic under the project Advanced Technologies for Heat and Electricity Production - TE01020036.

\section{References}

[1] 2013 ASHRAE Handbook-Fundamentals (SI), (ASHRAE, 2013)

[2] S. Herrmann, H. J. Kretzschmar, D. P. Gatley, Thermodynamical Properties of Real Moist Air, Dry Air, Steam, Water, and Ice, ASHRAE RP-1485, (ASHRAE, 2009)

[3] E. W. Lemmon, R. T. Jacobsen, S. G. Penoncello, D. G. Friend, J. Phys. Chem. Ref. Data, 29(3), (2000)

[4] Revised Release on the IAPWS Formulation 1995 for the Thermodynamic Properties of Ordinary Water Substance for General and Scientific Use, (IAPWS, 2014)

[5] K. Nerendra, B. Sudhamsa, B. M. Sarath, Research Journal of Chemical Sciences, 4(8), (2014), 42-45

[6] K. W. Woo, S. I. Yeo, The SNU Journal of Educational Research, 5, (1995), 127-134

[7] B. E. Poling, J. M. Prausnitz, J. P. O'Connell, The Properties of Gases and Liquids, (The McGraw-Hill Companies, 2001)

[8] L. Dvořák, J. Čížek, J. Nožička, EJP Web of Conferences, 92, (2015), 02014

[9] P. Vitkovič, EJP Web of Conferences, 114, (2016), 02137 


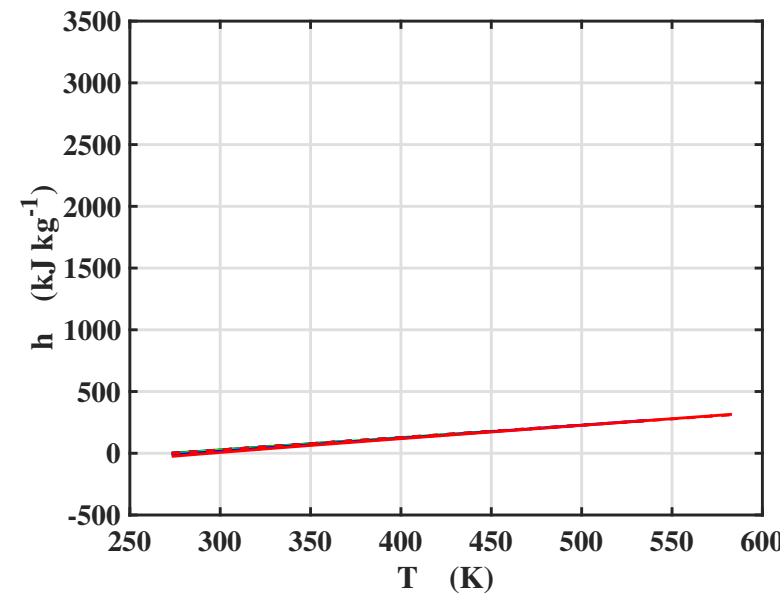

(a) Enthalpy, degree of saturation $0 \%$

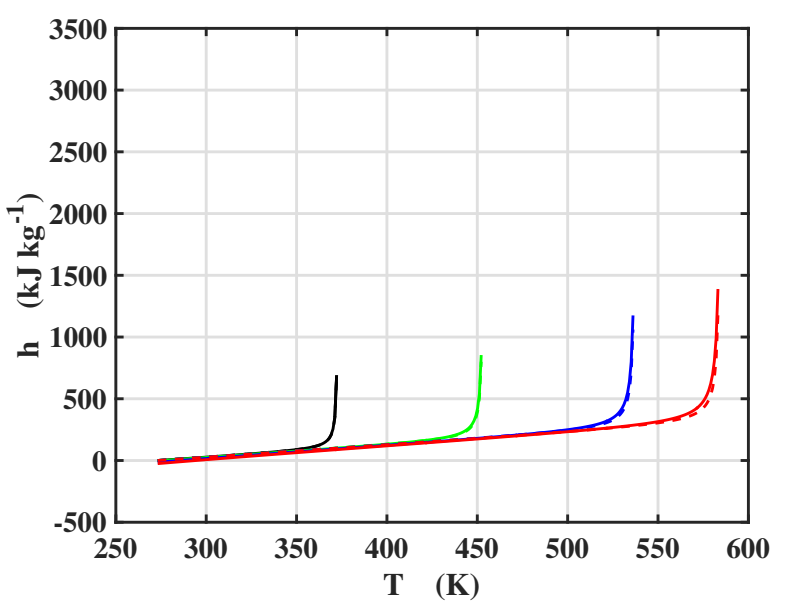

(c) Enthalpy, degree of saturation $1 \%$

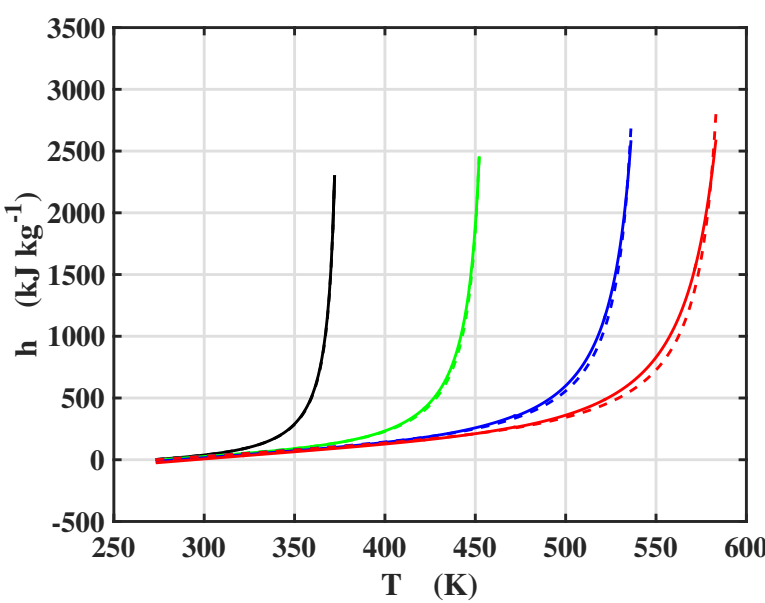

(e) Enthalpy, degree of saturation 20\%

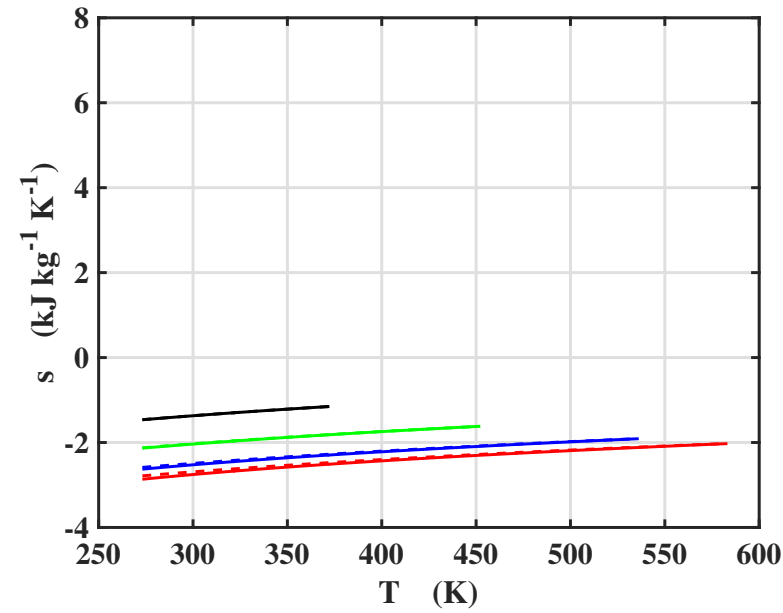

(b) Entropy, degree of saturation $0 \%$

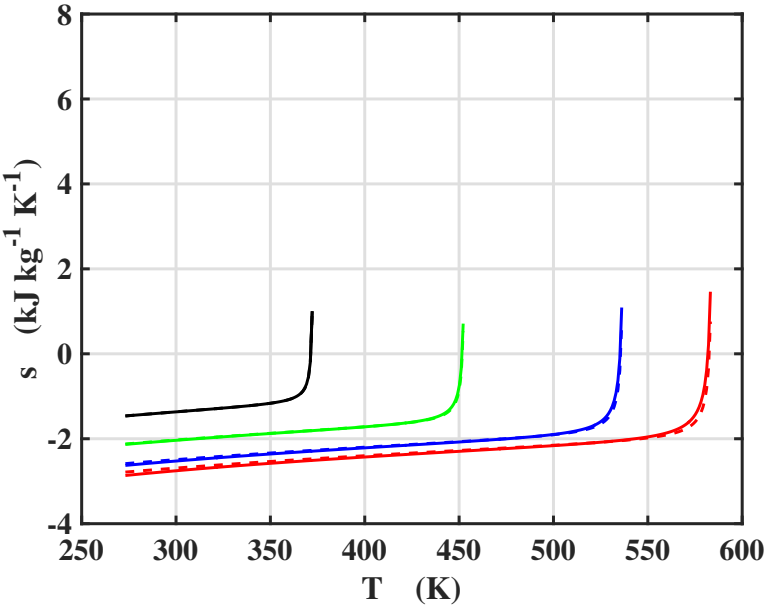

(d) Entropy, degree of saturation $1 \%$

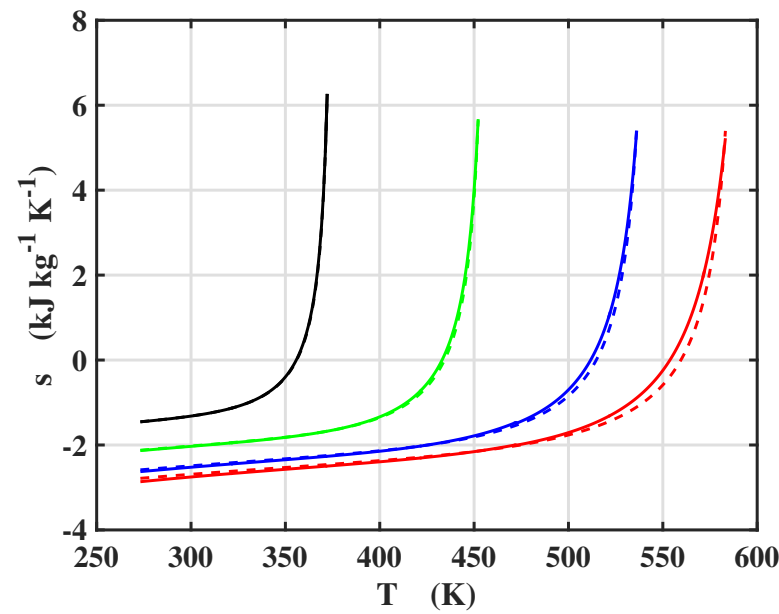

(f) Entropy, degree of saturation 20\%

Figure 1. Enthalpy and entropy of moist air as a function of pressure, temperature and degree of saturation; for pressure $0.1 \mathrm{MPa}$ (black), 1MPa (green), 5MPa (blue) and 10MPa (black); solid line represents engineering model and dashed line represents ideal gas model 


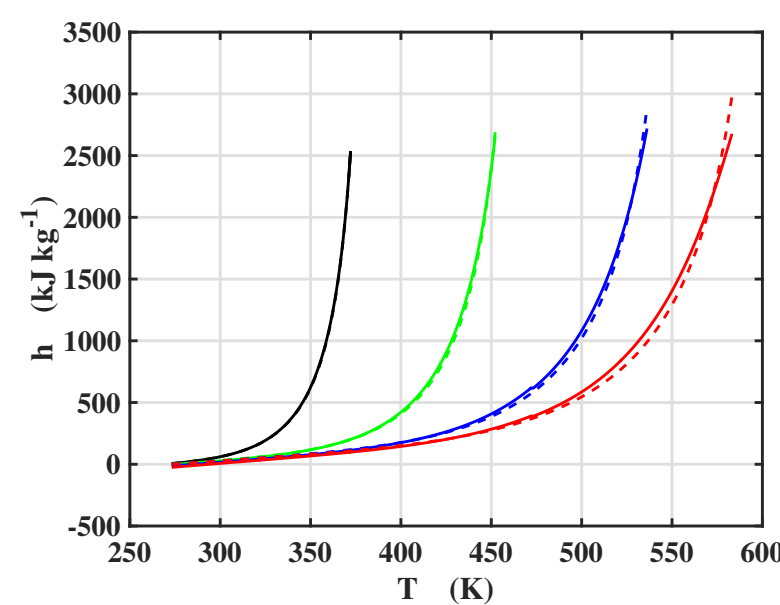

(a) Enthalpy, degree of saturation $60 \%$

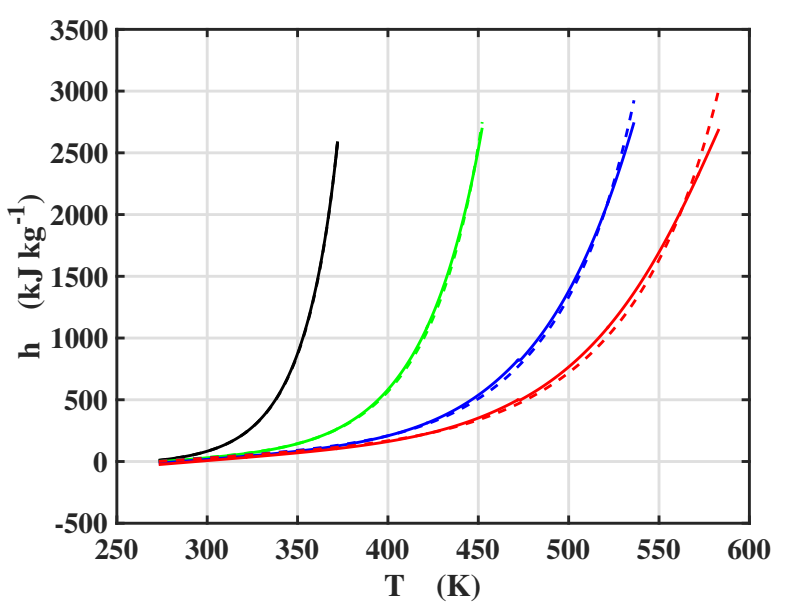

(c) Enthalpy, degree of saturation $100 \%$

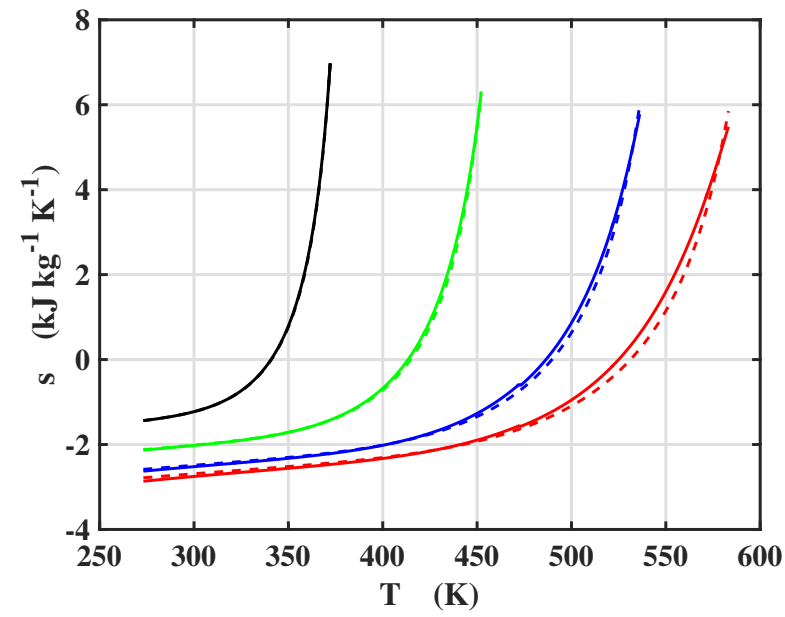

(b) Entropy, degree of saturation $60 \%$

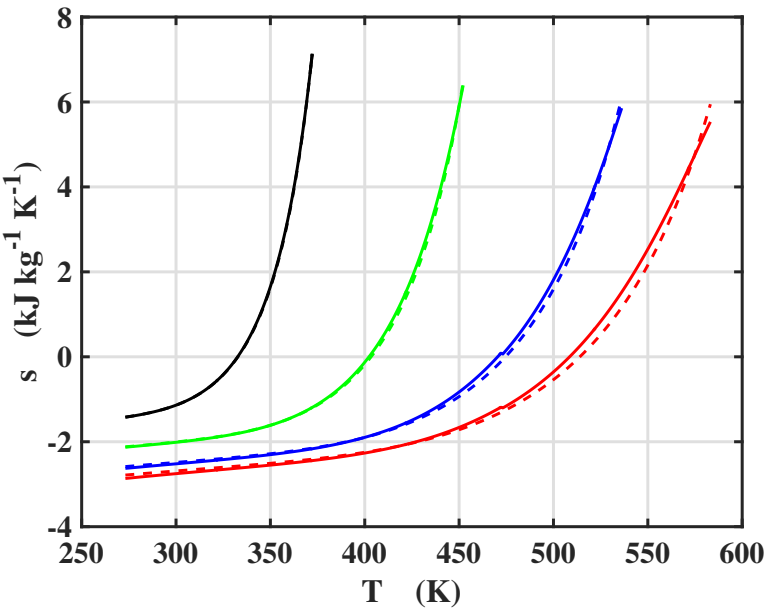

(d) Entropy, degree of saturation $100 \%$

Figure 2. Enthalpy and entropy of moist air as a function of pressure, temperature and degree of saturation; for pressure $0.1 \mathrm{MPa}$ (black), 1MPa (green), 5MPa (blue) and 10MPa (black); solid line represents engineering model and dashed line represents ideal gas model 


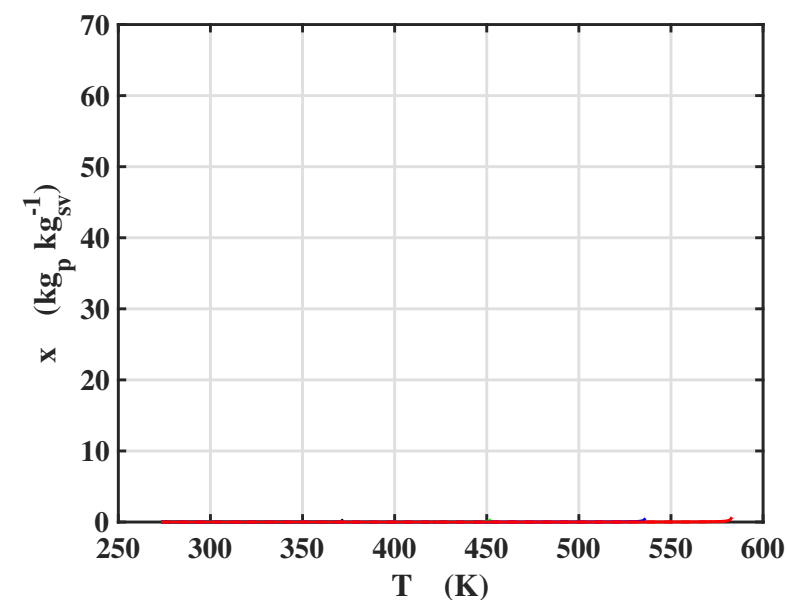

(a) Humidity ratio, degree of saturation $1 \%$

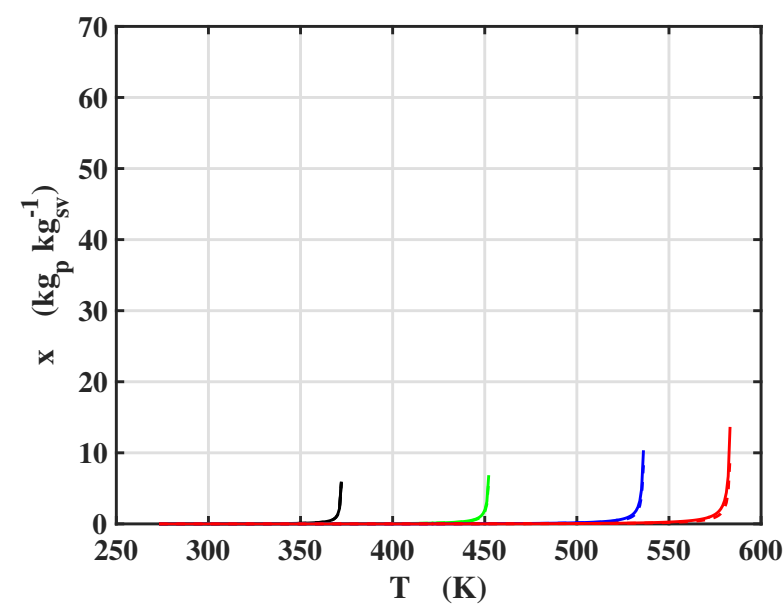

(c) Humidity ratio, degree of saturation $20 \%$

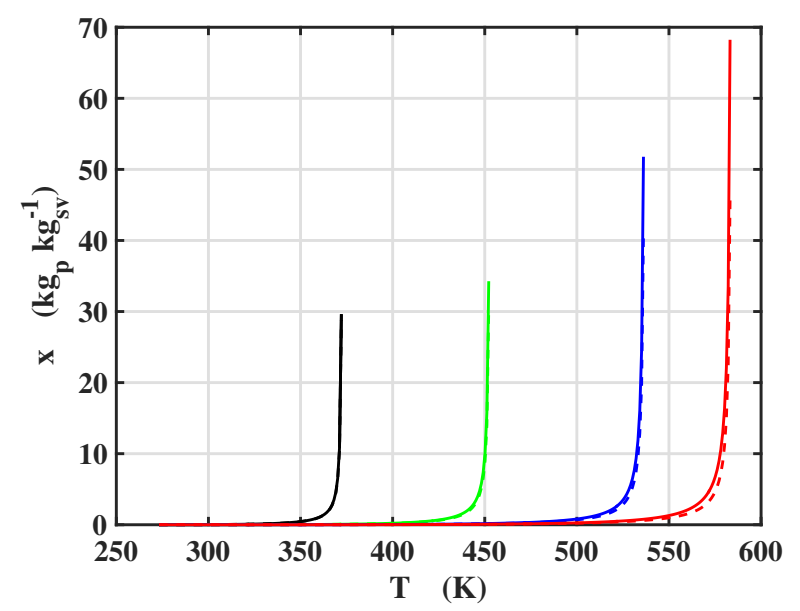

(e) Humidity ratio, degree of saturation $100 \%$

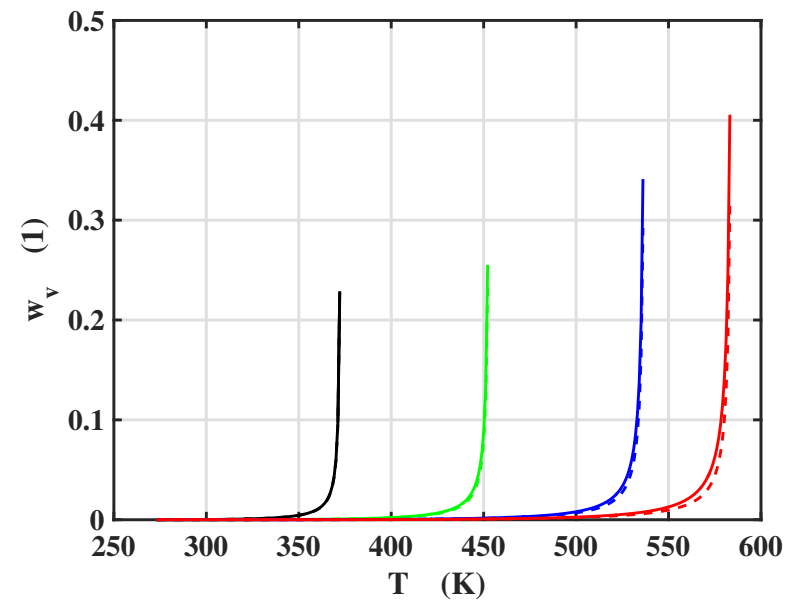

(b) Water vapour mass fraction, degree of saturation $1 \%$

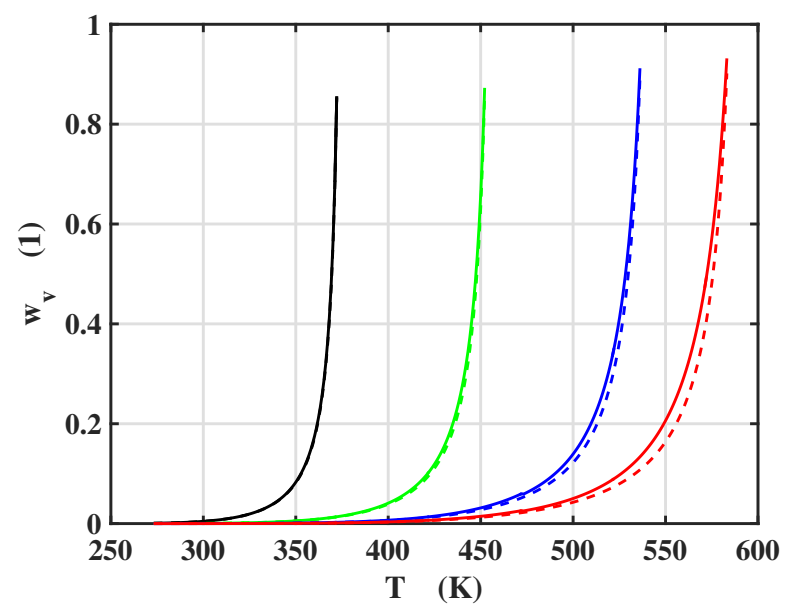

(d) Water vapour mass fraction, degree of saturation $20 \%$

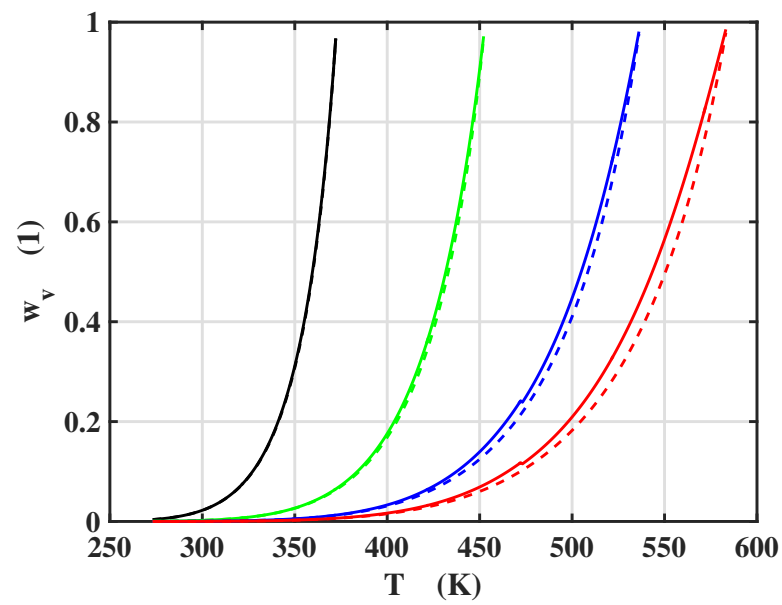

(f) Water vapour mass fraction, degree of saturation $100 \%$

Figure 3. Humidity ratio and water vapour mass fraction as a function of pressure, temperature and degree of saturation; for pressure $0.1 \mathrm{MPa}$ (black), $1 \mathrm{MPa}$ (green), 5MPa (blue) and 10MPa (black); solid line represents engineering model and dashed line represents ideal gas model 


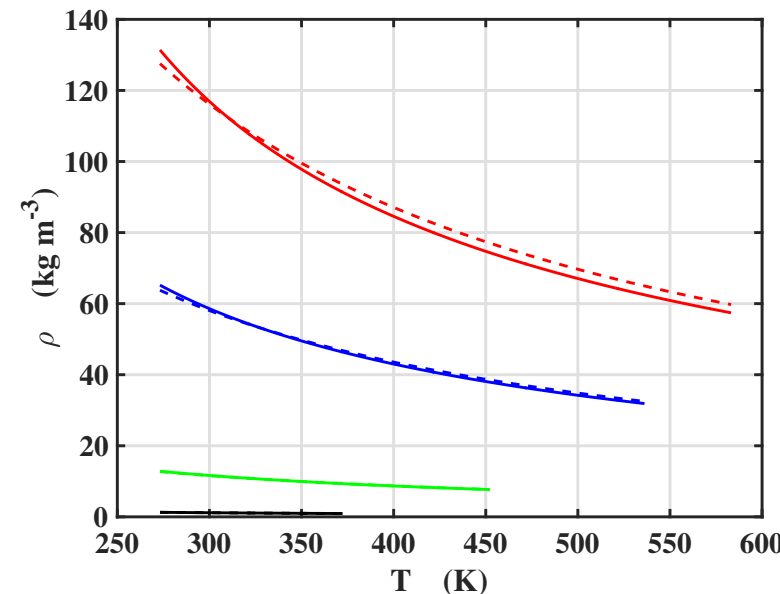

(a) Density, degree of saturation 0\%

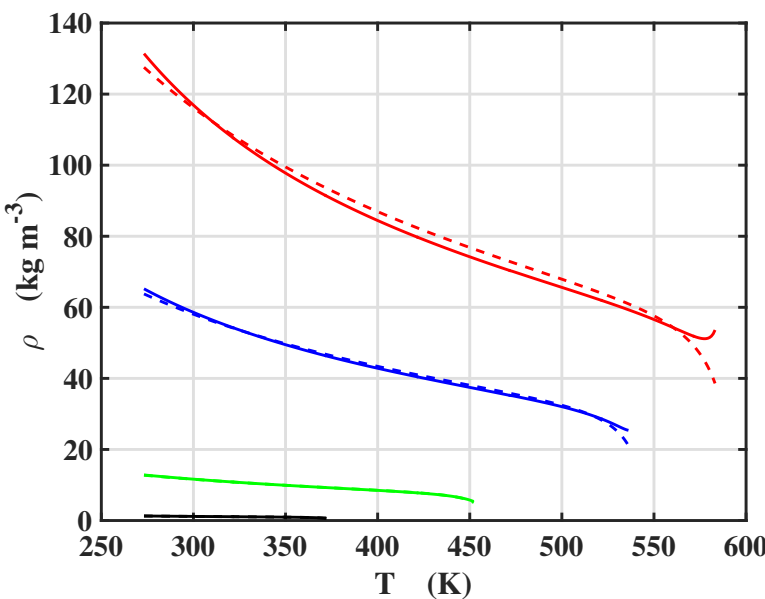

(c) Density, degree of saturation $20 \%$

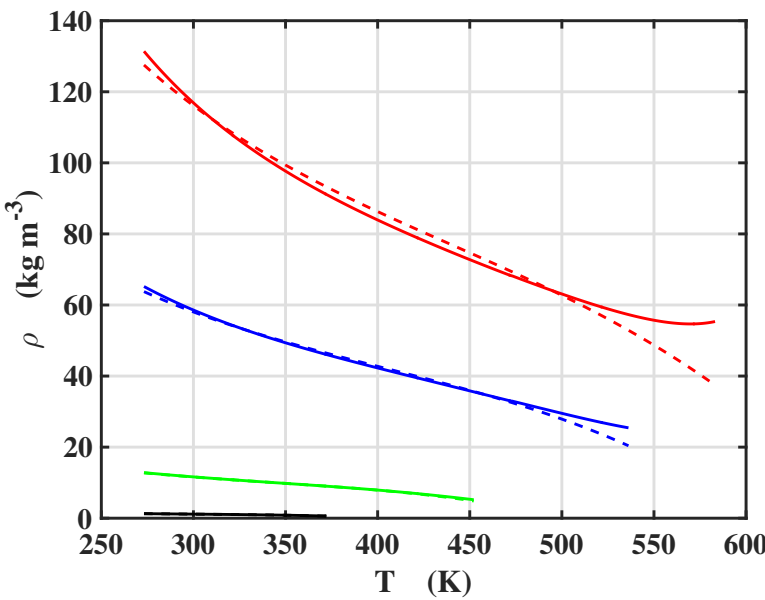

(e) Density, degree of saturation $100 \%$

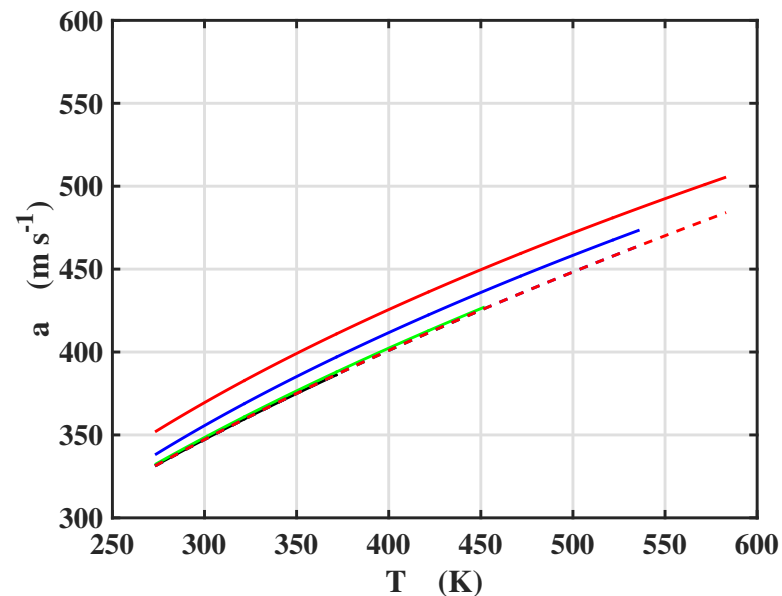

(b) Speed of sound, degree of saturation $0 \%$

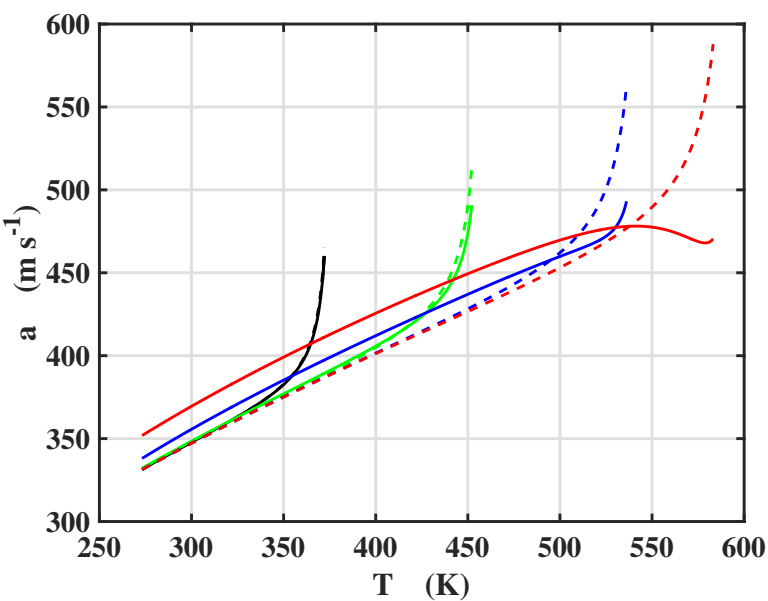

(d) Speed of sound, degree of saturation $20 \%$

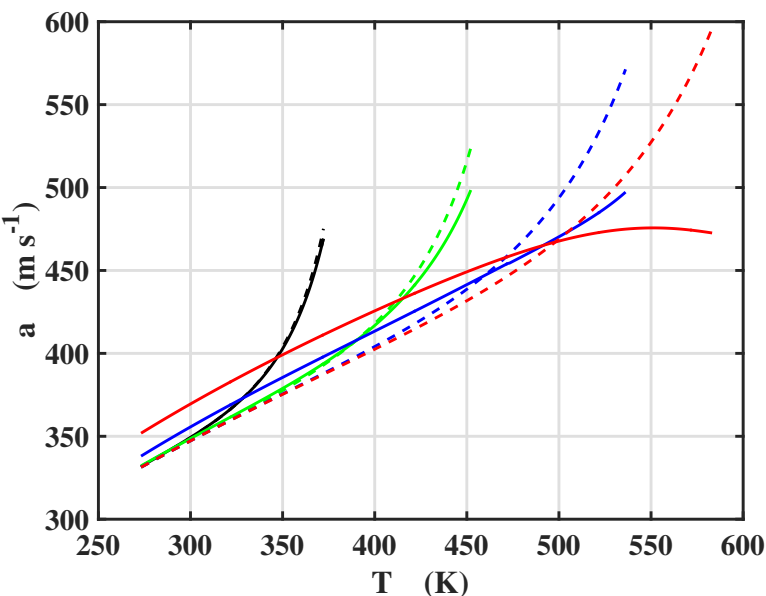

(f) Speed of sound, degree of saturation $100 \%$

Figure 4. Density and speed of sound of moist air as a function of pressure, temperature and degree of saturation; for pressure $0.1 \mathrm{MPa}$ (black), 1MPa (green), 5MPa (blue) and 10MPa (black); solid line represents engineering model and dashed line represents ideal gas model 